\title{
El rescate de las comunidades en el marco de la Globalización
}

\section{Vindicating communities in the context of Globalization}

\author{
Esther Wiesenfeld \\ Instituto de Psicología. Universidad Central de Venezuela \\ ewiesen@reacciun.ve
}

\section{Resumen}

La prevalencia, e incluso la agudización, en años recientes de problemas de distinta naturaleza (ambientales, sociales, económicos), particularmente en América Latina, ha conducido a diversos sectores de estos paises a cuestionar la globalización como modelo para su desarrollo.

En este artículo exponemos y analizamos, en términos generales, las concepciones, características e implicaciones de la globalización, desde dos versiones antagónicas acerca del fenómeno: la primera de ellas desde la concepción que sobre este proceso han construído sus gestores y partidarios y la segunda desde la versión elaborada por sus oponentes.

Al respecto, planteamos algunas similitudes existentes entre la primera versión con el paradigma dominante en la ciencia y entre la segunda versión con paradigmas emergentes. Así, en la primera versión la globalización se presenta como la única realidad posible, universal, irrefutable e irreversible, en tanto que en la segunda, se formulan distintas construcciones con relación a la globalización, que la significan como procesos cultural e historicamente constituídos y por tanto cambiantes.

Esta segunda versión es la que nos sirve como base para analizar el impacto de la globalización en comunidades en situación de desventaja económica en nuestro continente y a la vez para plantear las potencialidades de éstas, fortalecidas a través de las contribuciones de la psicología social comunitaria, para resistir los efectos desfavorables que sobre ellas tiene la globalización.

Palabras clave: Globalización; Pluralismo; Keywords: Globalization; Pluralism; Communities; Comunidades; Psicología social comunitaria

\begin{abstract}
The prevalence and increase, specially in recent years, of diverse problems (social, economic environmental) particularly in Latin America have led various sectors to question globalization as a convenient model for the development of these countries.
\end{abstract}

In this article we present and analyse, in general terms, some notions, characteristics and implications of globalization, from two antagonistic versions: The first of them refers to the notion of globalization from the point of view of its creators and adepts, while the second one is based on the version ellaborated by its oponents.

In this regard, we present some similarities between the first version with the dominant paradigm in science and between the second one with emergent paradigms. Thus, in the first version globalization is understood as the unique, universal, undeniable and irreversible reality, whereas in the second one various constructions regarding globalization, which signify this phenomenon as culturally and historically constructed and hence dynamic, are formulated.

This second version serves us as base for analysing the impact of globalization on economically deprived communities in our continent and simultaneously for illustrating the potentialities of these communities, empowered through the contributions of community social psychology, for resisting unfavourable effects of globalization upon them.

Community social psychology 


\section{¿Qué es la globalización?}

La globalización es un proceso concebido e interpretado de diferentes maneras, las cuales varían según quién, cómo y desde donde lo construye. Para sus gestores y adeptos se trata de un fenómeno universal, irreversible, cuya veracidad resulta incuestionable y que ha llegado para quedarse; mientras que sus adversarios cuestionan su universalidad, irreversibilidad y permanencia.

Si asumimos la existencia de los fenómenos, en este caso la globalización, como la única realidad posible, objetiva, independiente de nosotros y cuyo curso no podemos alterar, estaríamos posicionándonos en la primera concepción, la cual no deja de ser optimista para quienes se benefician de esta situación y fatalista para quienes la padecen. Por el contrario, si consideramos los fenómenos como construcciones sociales, históricamente constituídos y por lo tanto dinámicos, nos colocamos del lado de la segunda concepción.

De acuerdo con Daniel Mato (1999a, 2000a) la globalización en singular remite a un discurso sobre ésta como fetiche al que se le atribuye la causa o la solución de todos los problemas de la humanidad, cual fuerza suprahumana que actúa al margen de los actores y acciones sociales y por tanto anonimiza a los responsables de sus consecuencias. Propone en su lugar emplear el término procesos de globalización en plural, el cual, contrario al primero, incorpora los múltiples procesos asociados a los actores sociales con sus prácticas e interrelaciones complejas. Estas son, en definitiva, las que propician o impiden el desarrollo y/o consolidación de dichos procesos y las que les confieren una variedad de significados a los mismos.

Lo que los proponentes de ambas concepciones comparten es, que aunque la globalización no es reciente, por cuanto sus inicios se remontan a la conquista de América; en la actualidad se expresa de manera inédita, particularmente en lo que a las dimensiones económicas, tecnológicas y culturales respecta.

Las tendencias recientes de globalización se orientan hacia la integración del mercado y la subordinación a las necesidades del capital; la homogeneización social y cultural; la desestructuración de la industria cultural endógena; el monopolio de los medios de comunicación; la libertad individual en menoscabo de la participación colectiva; la pérdida de garantías laborales y de seguridad social de los trabajadores y sindicatos; la pérdida de legitimidad del estado nación como ente soberano, sometido ahora al poder transnacional y a nuevas regulaciones para el desarrollo del comercio; la supresión de sus derechos sobre su propio espacio (Chomsky, 1995; Garrido, 1995; García Canclini, 1997; Caponi y Mendoza, 1997; Serbín, 1997) entre otros.

Hay quienes sostienen sostienen que este desmantelamiento del Estado está desmembrando las identidades nacionales y provocando nuevas formas de violaciones de los derechos humanos universales en cuanto a salud, educación, vivienda, alimentación, trabajo, debido a la enorme desigualdad social que acarrea (Monsonyi, 1997; Fariñas, 2000).

Estas tendencias coexisten con movimientos contrarios a ellas, generándose tensiones entre el universalismo individualista, orientado a la acumulación de capital y el pluralismo de las identidades, entre el tránsito de lo local a lo global y viceversa. Al decir de Bonaventura Dos Santos (citado en Fariñas, 2000) la relación entre lo local y lo global se puede visualizar como las dos caras de una misma moneda, en la que los fenómenos locales son globalizados y a su vez las políticas transnacionales impactan en las condiciones locales, es decir a etnias, religiones, tribus, naciones. 
Por su parte, Nestor García Canclini (1999) plantea la imposibilidad de la existencia de lo global sin lo local, sin embargo cuestiona la forma de ver la relación entre lo local y lo global, cual relación entre opuestos y de ubicar el debate en los desencuentros entre las políticas de integración supranacional y la conducta ciudadana. Propone más bien incluir las mediaciones existentes entre ambos extremos, de modo de facilitar interconexiones entre actores que potencien las iniciativas sociales, legitimen las instituciones y los motiven a negociar la diversidad desde una confrontación dialógica y desde la coexistencia de diferentes concepciones. Para este autor ello supone la apertura hacia diversas experiencias culturales, es decir a la globalización desde la diferencia o al cosmopolitismo de la globalización. Se trata, entonces, de un fenómeno pluridimensional, pluridisciplinar, polisémico y complejo, que ha incidido en las vidas de todos los seres humanos.

\section{¿Cuáles son algunos de los efectos y paradojas de los procesos de globalización?}

Los defensores de los procesos de globalización consideran que éstos constituyen un medio para ayudar a los países, particularmente los pobres, a progresar económicamente mediante su integración en el sistema de mercado internacional; que favorecen la expansión del conocimiento al promover el intercambio de saberes de distintas culturas y que estimulan la solidaridad internacional, entre otros (Adamson, 2000; Casals, 2001; Reed, 1996).

Por su parte los críticos de la globalización objetan esta visión positiva con base en las siguientes argumentaciones:

a. La liberalización comercial y el aumento de la competencia de los mercados internacionales, los cuales se colocan en lugares donde la mano de obra es más barata, han provocado el colapso de pequeñas y medianas industrias, con el consecuente despido de trabajadores, quienes no sólo pierden su empleo, sino también los beneficios sociales asociados a éste.

b. La flexibilización del mercado laboral, como estrategia para aumentar la confianza de los inversores, ha atentado contra la estabilidad laboral, en la medida que las reglas de contrato han despojado a los trabajadores de toda posibilidad de resistencia al verse obligados a escoger entre un empleo efímero o el desempleo.

c. Los nuevos desempleados, pertenecientes fundamentalmente a estratos socioeconómicos medios y bajos, han intentado resolver su sustento mediante el empleo informal, la migración, o aumentando las presiones sobre la ya deteriorada infraestructura, como ultimo recurso de subsistencia. Su empobrecimiento ha debilitado además su capacidad para sostener las instituciones propias de la sociedad civil y por ende la toma de decisiones que les compete se ha visto mermada (Reed, 1996).

d. El impacto de la eliminación de fronteras para el libre comercio no ha sido simétrico. Mientras que para algunos ha sido la expresión de libertad de movimiento, de búsqueda de placer en diversos confines del mundo, las fronteras se han cerrado para quienes escogen la migración como un recurso para la supervivencia, no obstante los riesgos que la misma acarrea para la propia vida.

La eliminación de fronteras también ha estimulado la supresión del lugar como escenario de relaciones, de experiencias, lo cual invisibiliza a los actores de ese lugar y a sus prácticas. Es así como el espacio ha reemplazado al lugar y se ha convertido en el no lugar con el cual no se pueden establecer identidades ni afectos, pero que reafirma la distribución social y económica asimétrica de ese espacio (Escobar, 2001). 
e. La agricultura a pequeña escala se ha visto reemplazada por la automatización de la agricultura corporativa y biotecnológica y por una privatizacion de los espacios abiertos de la tierra. En consecuencia, la permanencia de los pobladores en sus lugares de origen también se ha visto amenazada por cuanto al despojarlos de las tierras que por años les sirvieron como albergue y sustento, las empresas privadas los despojaron de proyectos, memorias y valores de convivencia comunitaria allí construidos (Rivadeneyra, 1997; Monsonyi, 1997). A ello se añade que muchas de estas empresas han explotado las tierras provocando graves daños al entorno.

Ejemplo de ello es el reporte de la organización norteamericana de derechos ciudadanos "Public Citizen", el cual revela que la Organización Mundial de Comercio (OMC) ha denunciado la política de reducción de las emisiones contaminantes de carburantes por atentar contra intereses de los productores de gasolina. (Casals, 2001) o el Protocolo de Kyoto no suscrito por Estados Unidos por considerar que atenta contra el crecimiento económico de dicho país.

Con base en los efectos expuestos, sorprende la advertencia que la supervivencia de los países del Tercer Mundo depende de su participación incondicional en el mercado global, toda vez que el crecimiento económico, impulsado por los programas de ajuste estructural y por la economía neoliberal, no ha disminuido la pobreza, los problemas de desempleo, superpoblación, desigualdad social, exclusión, vivienda, hambre, analfabetismo, educación, salud, injusticia, la inseguridad personal, deterioro ambiental. Incluso las distintas Cumbres Sociales han admitido el fracaso del modelo económico en resolver los problemas mencionados (Rogalski, 1997; Rivadeneyra, 1997).

Algunos datos que ilustran esta afirmación son los siguientes: 800 millones de personas padecen de desnutrición y 4000 millones viven en estado de pobreza, la riqueza de las 225 personas más ricas del mundo representa la renta del $47 \%$ de los más pobres del mundo, o sea que 225 personas ganan lo que 2.500 millones (Casals, 2001). La contribución anual del 1\% de esta riqueza podría suplir de educación primaria a todo el mundo (Adamson, 2001). Algunos han llegado a afirmar que la globalización no es tan global ya que geográficamente se desarrolla fundamentalmente en tres focos de la economía mundial lo que deja fuera continentes enteros y al $70 \%$ de la población mundial (García Morales, 1996; Ridadeneyra, 1997; Rogalski, 1997).

Al parecer la promesa de un mundo mejor, cuya consecución va precedida de una etapa de sacrificios, mientras se alcanza el progreso económico, no se ha cumplido, al menos para la mayoría. Por el contrario, se ha traducido en una brecha cada vez mayor entre los que disfrutan de la satisfacción de necesidades que no tienen y los que tienen necesidades casi imposibles de satisfacer. No en vano se ha calificado la era de la globalización como la era del consumo, al consumismo como la nueva forma de ciudadanía (García Canclini, 1995, 1999) y al ciudadano como "homo economicus", como agente de producción, intercambio y consumo de mercancía con miras a maximizar el beneficio económico (Monsonyi, 1997). Tal como lo expresa Zygmunt Bauman (1999: 109): este tipo de sociedad "no es de aprendizaje sino principalmente de olvido, cuando se despoja el deseo de la demora y la demora del deseo, la capacidad de consumo se puede extender mucho más allá de los límites impuestos por las necesidades naturales o adquiridas del consumidor".

Evidentemente existe una discrepancia entre el discurso globalizador y las prácticas globalizadoras, y sucede que tales prácticas y sus efectos no favorecen a la mayoría de la población mundial. 


\section{¿Es posible una globalización diferente?}

Las diferentes maneras de interpretar la globalización y sus efectos, se relacionan estrechamente con los diversos modos de reaccionar ante ella. Quienes la consideran como la panacea universal la defienden; para otros no es la panacea pero la asumen como inevitable y por tanto no ven sentido en desafiarla; un tercer grupo la responsabiliza de los problemas de la humanidad, o parte de ellos y plantea combatirla; un cuarto sector, menos radical que el anterior, vislumbra posibilidades de volcarla al servicio de la humanidad, de aprovechar las oportunidades que brinda para el enriquecimiento cultural, compromiso político, justicia económica. En fin, defensa, resignación, resistencia o propuestas de globalización alternativas; son algunas formas de enfrentar los procesos de globalización

La defensa y la resignación ante la globalización, en tanto fuerza supranatural, producto de factores financieros y/o tecnológicos inevitables o resultado de la voluntad de un pequeño pero poderoso número de gobernantes (García Canclini, 1999; Mato, 2000a; Añón, 2000), contribuyen a ignorar los actores y sus prácticas sociales y por ende a paralizarlos y alienarlos (Mato, 2000b). Al decir de Michel Rogalski (1977), promueve la globalización que hemos permitido.

Esta globalización, en singular ( $y$ sin pretender elevarla al status de paradigma), comparte características similares a las atribuídas al paradigma científico dominante, el positivismo lógico. Así: a) su definición como entidad universal, única, descontextualizada, sin límites espaciales ni temporales, que desterritorializa y anonimiza a los actores y sus prácticas recuerda la ontología realista de dicho paradigma; b) el poder conferido a y asumido por sus gestores, quienes saben y deciden qué conviene a quién, dónde y cómo, y su desdibujamiento frente a los supuestos beneficiarios de sus políticas, asemeja a la distancia y neutralidad que adopta el/la investigador/a qua experto/a frente a los sujetos, que constituyen su objeto de conocimiento. Esa distancia y la dicotomía que impone en la relación gestor/a o investigador/a y sujetos coincide con la epistemología dualista del paradigma positivista.

Por el contrario, a) asumir la globalización como un programa impulsado por un conjunto de agentes que responden a sus concepciones (imaginarios parafraseando a García Canclini o representaciones sociales en términos de Mato, 1999b) de la globalización, y b) concebir tales concepciones como una de sus tantas versiones posibles, abre la posibilidad de crear y difundir otros imaginarios, narrativas, metáforas o construcciones, de la globalización, sustentados sobre la base de las experiencias de los diversos actores en sus respectivos lugares y de combatirla o de generar propuestas y acciones alternativas.

Estas dos orientaciones, es decir concebir la globalización como proceso que involucra diferentes actores y admitir que los discursos de estos construyen socialmente este fenómeno y por tanto inciden en las posibilidades que tiene de manifestarse, coinciden con paradigmas y metodologías críticas, formulados principalmente desde las ciencias sociales. Estos, sin referirse necesariamente a los procesos de globalización, constituyen formas de cuestionamiento o deconstrucción de aquellos. Me explico. El origen de estos paradigmas (construccionismo social, feminismo, psicología social crítica) y metodologías (metodología cualitativa, investigación acción participativa) está marcado por una oposición a la desigualdad y a la dominación en todos sus órdenes y como manifestación de ello estimulan acciones orientadas a promover la libertad, la emancipación, el bienestar social y a reivindicar los derechos de todos los ciudadanos a una vida digna, asignándoles un papel protagónico en la implementación de aquellas. 
En este orden de ideas, acciones tales como impulsar el fortalecimiento de agrupaciones en la sociedad; fomentar reflexiones críticas acerca de las propias prácticas e interacciones sociales de sus integrantes, articuladas en discursos construídos colectivamente; respetar y difundir estos discursos y desarrollar acciones transformadoras y/o reivindicativas orientadas por estos, son estrategias coherentes con la agenda de estas perspectivas.

El fortalecimiento de las agrupaciones a las que nos referimos ha incluido, entre otros, el rescate de las comunidades, contexto que ha resistido históricamente todo tipo de transformaciones geopolíticas (Tourraine, 1997); la construcción y movilización de una sociedad civil heterogénea, transnacional, orientada a recuperar los derechos ciudadanos y su participación en la toma de decisiones de asuntos que les conciernen (García Canclini, 1999); la reivindicación del lugar como contexto cuyas particularidades posibilitan tales acciones.

Para cumplir con este propósito han sido claves el desarrollo de perspectivas teóricas, metodológicas y epistemológicas centradas en las prácticas de los actores sociales, que propician la construcción y difusión de narrativas alternativas, en nuestro caso sobre la globalización y contrarrestan las de otros agentes que atentan contra sus intereses y de la docencia y la investigación orientadas por estas perspectivas (Mato, 1999b, 2000b; Lander, 2001).

El rescate de las comunidades locales incorpora la valoración del conocimiento popular y científico local, el refuerzo de la identidad comunitaria y de lugar y su defensa frente a modelos hegemónicos que invisibilizan a sus actores y que pretenden imponerse como universales y absolutos (Marsella, 1998; Agudo, 2001; Wiesenfeld, 2000). Las comunidades, en general, han sido efectivas como ámbito de resistencia a procesos de dominación, ya que su origen y desarrollo se fundamenta en la existencia de intereses compartidos, de acciones conjuntas para atenderlos, de vínculos socioafectivos consolidados históricamente a través de sus interacciones, todo lo cual permite anticipar la continuidad de sus acciones en el tiempo.

Por su parte, la construcción de una sociedad civil plural en los términos arriba planteados, requiere saber cómo podemos ser y hacer con los otros, cómo encarar las divergencias, lo cual a diferencia de la resistencia local, no se cierra sobre sus protagonistas y por tanto constituye, de acuerdo con García Canclini (1999) una resistencia más potente a la globalización que la que pueden promover las comunidades al replegarse para defender su identidad, su territorio, su religión.

Así, la Declaración de Iquitos, (Amazonia peruana) en mayo de 1990 en la que participaron organizaciones no gubernamentales transnacionales y la Coordinadora de Organizaciones Indígenas de la Cuenca Amazónica, COICA, planteó la alianza entre sectores indígenas y ambientalistas para el logro de una "Amazonia para la humanidad" en respuesta al grave deterioro de la biosfera. También reivindicó el llamado a una "Amazonia indígena" como símbolo del reconocimiento de los territorios indígenas para que dichos pueblos desarrollaran programas de conservación ambiental. Esa doble caracterización expresa las convergencias y divergencias entre las dos partes de la alianza (Mato, 1999b) A ambas subyace la idea que la Amazonia no compete a los estados ni a otros centros de las sociedades nacionales sino a alianzas entre actores tanto globales como locales que optaron por organizarse y establecer relaciones transnacionales para defender lo poco que les queda, debido a las acciones negligentes de los gobiernos nacionales. Dicen que para que sus gobiernos aceptaran lo que ellos llevaban años pidiendo las peticiones tuvieron que ser dichas en ingles por representantes de los organismos transnacionales. 
Vale la pena destacar que la potenciación de las agrupaciones mencionadas se ve impulsada por los propios procesos de globalización, los cuales al atentar contra posesiones, arraigos y lealtades de distinta naturaleza, e intentar erradicar prácticas que forman parte del mundo de vida de los grupos en cuestión, fomentan en las personas cuyas afiliaciones y pertenencias se ven amenazadas, la defensa y el fortalecimiento de sus vínculos, de sus lugares, de sus esferas de contención. De modo que la presión hacia la homogeneización, hacia el anonimato, hacia la invisibilidad, hacia el no lugar, ha propiciado paradójicamente sus contrarios, esto es la defensa de la heterogeneidad, de la identidad, de la visibilidad, del lugar y con ello un repunte de las agrupaciones, una apropiación del espacio público como espacio colectivo para el ejercicio de la ciudadanía. Al decir de Tourraine (1997), una recomunitarización.

Adicionalmente y contrario a la postura que ignora el lugar como categoría analítica relevante frente a los procesos de desterritorialización que promueve la globalización, el afianzamiento de las agrupaciones ha tenido como sustrato fundamental esta dimensión. Como sabemos, tanto las comunidades locales como la sociedad civil están compuestas por personas cuyas acciones ocurren en escenarios particulares, esto es, en lugares, de modo que los tres, personas, lugares y procesos, conforman una totalidad no susceptible de ser fragmentada. El lugar es entendido desde este marco interpretativo, no como mundo material objetivo, neutro, casual y sustituíble, sino como realidad subjetiva que forma parte de las experiencias humanas, inseparable del mundo de vida de las personas, de sus experiencias y significados.

Al respecto Edward Sampson (1989), en un artículo en el que plantea la necesidad de una nueva teoría para la psicología, que responda a los cambios y a las demandas producidas por la era de la globalización, propone una perspectiva constitutiva del ser humano, desde la cual no tiene sentido hablar de personas abstrayéndolas de sus comunidades particulares, porque estas son aspectos esenciales de sus identidades personales. En su opinión, los apegos a las comunidades no describen atributos de las identidades de las personas, sino que son constituyentes de tales identidades. Los aportes de autores socioconstruccionistas como Kennet Gergen, Tomás Ibañez, Yvonna Lincoln y Egon Guba, entre otros, son fundamentales para esta reconceptualización de la psicología y de su objeto de estudio.

Las orientaciones paradigmáticas y metodológicas mencionadas constituyen herramientas que favorecen la realización de las actividades sugeridas y la consecución de los logros formulados. Nos referimos principalmente a la reivindicación de las comunidades locales amenazadas por los procesos de globalización, a través de prácticas sociales que las visibilicen y reafirmen en sus particularidades, las cuales, como dijimos antes, se refuerzan y enriquecen en su apertura al intercambio con otros actores, procesos y lugares.

Las universidades, como centros docentes y de investigación, pueden y deben jugar un papel importante en la formación de recursos humanos preocupados por los procesos de globalización, y por ende interesados y capacitados en el desarrollo de investigaciones orientadas a comprender los diferentes significados de estos procesos, a propiciar en distintos sectores una reflexión crítica acerca de ellos, a promover procesos identitarios y acciones reivindicativas y transformadoras de las condiciones sociales opresoras.

Muchas acciones ya están en marcha. Están en marcha acciones de minorías étnicas, sectores ecologistas y de defensa de los consumidores, vanguardias laborales, pensadores críticos, movimientos antiglobalización (Rivadeneyra, 1997). Estas acciones han impactado a la opinión 
pública, a los medios de comunicación, a las organizaciones financieras (Serbin, 1997). Sin embargo muchas de ellas no trascienden acciones puntuales que no promueven los procesos psicosociales necesarios para una resistencia sustentable.

En este particular la Psicología Social Comunitaria (PSC) tiene mucho que aportar.

\section{¿Cómo puede contribuir la PSC a enfrentar los efectos perversos de la globalización?}

Partimos de la premisa que la PSC cumple con los requerimientos para ser considerada como disciplina de resistencia a la globalización.

¿Sobre qué argumentos sustentamos esta premisa?

En primer lugar, consideramos que los antecedentes, características, fundamentos teóricos y estrategias metodológicas de la PSC son compatibles con la noción de globalización en plural, es decir como procesos, socialmente construídos, inadmisibles en su versión hegemónica y por lo tanto provisionales, alterables y perfectibles.

En segundo lugar, el nacimiento de la PSC constituye en sí un acto de rebeldía frente a un paradigma hegemónico, orientado a la preservación del status quo, con una concepción de sujeto cual objeto o realidad externa, al cual el investigador se aproxima de manera distante y neutra, con un abordaje metodológico que garantiza la obtención de un conocimiento válido y confiable, descontextualizado y ahistórico sobre este sujeto objetivado. En rechazo a esta perspectiva, la PSC nace con un compromiso hacia el cambio social que contribuya a mejorar las condiciones de vida de los sectores económicamente desfavorecidos de la sociedad.

Para ello se basa en un conjunto de fundamentos ontológicos y epistemológicos, asociados fundamentalmente al socioconstruccionismo y a la teoría crítica, y metodológicos, principalmente la Investigación Acción Participativa (IAP) y principios derivados de la educación popular como la problematización y la concientización. Estos fundamentos: a) comparten una noción de las comunidades como entidades dinámicas, gestoras de su propio destino, cuyos conocimientos y experiencias merecen ser escuchados y difundidos, al igual que lo son los saberes privilegiados por la ciencia; b) las realidades de estos sujetos no son únicas ni independientes de ellos, sino múltiples, históricas, construídas socialmente en sus interacciones sociales y vinculadas al contexto particular en el que ocurren; c) conciben al profesional como facilitador y copartícipe de procesos orientados al cambio; d) postulan una relación simétrica, horizontal, de respeto mutuo entre el profesional y los sujetos; relación en la que ambos se nutren y transforman; comprometen sus afectos, sus saberes, sus experiencias, y orientan sus acciones hacia el cambio social antes mencionado; e) enfatizan la unión entre teoría y praxis, de modo que la teoría se construye a partir de la reflexión crítica de la acción (concientización), y conforma un tipo de saber que articula el conocimiento científico con el popular. Este ciclo de reflexión-acción-reflexión, se repite indefinidamente en forma de espiral, de modo que en cada ciclo la reflexión sobre la acción aumenta la complejidad del conocimiento obtenido y aplicado; f) asimismo consideran la investigación y la acción como inseparables; el interés se centra tanto en la producción del conocimiento como en el cambio social. 
El cambio al que se refiere la PSC es concebido e implementado conjuntamente con los involucrados a partir de la reflexión crítica colectiva acerca de condiciones sociales adversas percibidas como naturales (problematización) lo cual permite llegar a entenderlas como productos históricos de condiciones de explotación alterables a través del esfuerzo conjunto (concientización).

Estos procedimientos favorecen la producción y circulación de múltiples narrativas con relación a las experiencias vividas y orientan la formulación y desarrollo de acciones reivindicativas y transformadoras, basadas en la organización y participación comunitarias. Los logros derivados de tales acciones son múltiples. Por una parte empoderan a sus protagonistas, aumentando su autonomía y su confianza, su poder y su control sobre sus vidas, fortalecen su sentido de comunidad, de identidad y de apego con las personas y el lugar. Por otro lado, la resignificación o producción de nuevas narrativas, desafía discursos hegemónicos, como el de la globalización y los efectos perversos que lo acompañan (Mato, 1999b). En efecto la expresión "la globalización ha llegado para quedarse", sólo puede deconstruirse con narrativas diferentes que desmitifiquen la irreversibilidad, permanencia o modos de constituirse la globalización.

Las estrategias empleadas por la PSC apuntan hacia la producción y difusión de versiones alternativas de la realidad y hacia la incorporación de voces cuyas narrativas son usualmente ignoradas. La comunidad, espacio privilegiado por esta disciplina constituye un ámbito idóneo para promover estrategias como las señaladas, ya que su permanencia en el tiempo y en el lugar, constituyen garante de la sostenibilidad de las relaciones y acciones de sus miembros.

Entonces ante las preguntas formuladas en este trabajo: ¿Es posible una globalización diferente? ¿Cómo puede contribuir la psicología social comunitaria a resistir la globalización?, podemos responder con una cita de Octavio lani (1999):

"Los pueblos de todos los continentes han sido capaces de movilizar elementos obtenidos de los pueblos colonizadores para desarrollar sus perspectivas de autoafirmación, autoconciencia y lucha, a veces con limitaciones y otras con sorprendentes invenciones." (Iani, 1999: 30).

Esto mismo podríamos decir de las experiencias derivadas del trabajo psicosocial comunitario. Con esto no queremos sugerir que la PSC tiene la clave para la solución de los problemas derivados de los procesos globalizadores, pero sí podemos afirmar, que sus herramientas y los valores que orientan la acción de los profesionales involucrados en el quehacer psicosocial comunitario, constituyen un baluarte importante para resistir o al menos aminorar los efectos de este y otros procesos de dominación psicológica, social, política, económica y académica.

\section{Referencias}

Adamson, Gladys. (2000). Psicología social y globalización. El papel de la psicología social en Latinoamérica en los albores del Tercer Milenio. Conferencia abierta dictada en la Universidad Luis Amigo, Medellin, Colombia. 12 de mayo de 2000.

Agudo, Ximena. (2000). Identidad, lugar y poder: Un marco interpretativo en expansión. Revista del Instituto Universitario Pedagógico, 4, (2) 107-130. 
Añon, María José. (2000). El test de la inclusión: Los derechos sociales. En Antonio Antón. (Coord). Trabajo, derechos sociales y globalización. Algunos retos para el siglo XXI (pp. 148-191). Madrid: Talasa.

Bauman, Zygmunt. (1999). La globalización. Consecuencias humanas. México D.F: Fondo de Cultura Económica.

Caponi, Orietta y Mendoza, Humberto. (1997). La filosofia neoliberal y su concreción en las políticas públicas de Venezuela. Nuevo Sur, 147, 46-56.

Cariola, Cecilia y Lacabana, Miguel. (2000). Transformaciones en el trabajo, diferenciación social y transformación de la metrópoli: el Área Metropolitana de Caracas. Cuadernos del CENDES, 17 (43), 85-120.

Casals, Carles. (2001). Globalización: Apuntes de un proceso que está transformando nuestras vidas. Barcelona: Intermon.

Escobar, Arturo. (2000). El lugar de la naturaleza y la naturaleza del lugar: ¿Globalización o postdesarrollo?. En Edgardo Lander (ed.). La colonialidad del saber: eurocentrismo y ciencias sociales. Perspectivas latinoamericanas. Caracas: UNESCO, Ediciones FACES/UCV.

Fariñas, María José. (2000). Globalización, ciudadanía y derechos humanos. Cuadernos Bartolomé de las casas. Madrid: Dykinson.

García Canclini, Nestor. (1995). Consumidores y ciudadanos: Conflictos multiculturales de la globalización. México D.F: Grijalbo.

García Canclini, Nestor. (1999). La globalización imaginada. México D.F: Paidos

Garcia Morales, Federico. (1996). Identidad y Globalización. Las alternativas en un mundo en crisis. Ponencia presentada ante el $\mathrm{V}$ Congreso de la Asociación Mexicana de Estudios Internacionales (AMEI) Oaxtepex, México. 30 de noviembre de 1996.

Garrido, Luis Javier. (1995). La crítica del liberalismo realmente existente. En Noam Chomsky y Heinz Dieterich (Comp.). La sociedad global. Educación mercadeo y democracia (pp. 7-14). México D.F: Contrapuntos.

Ianni, Octavio. (1999). La era del globalismo. México D.F: Siglo Veintiuno.

Marsella, Anthony. (1998). Toward a global psychology: Meeting the needs of a changing world. American Psychologist, 53, 1282-1291.

Mato, Daniel. (1999a). Sobre la fetichización de la "globalización" y las dificultades que plantea para el estudio de las transformaciones sociales contemporáneas. Revista Venezolana de Análisis de Coyuntura, V (1), 129-147.

Mato, Daniel. (1999b). Globalización representaciones sociales y transformaciones sociopolíticas. Nueva Sociedad, 163, 152-163

Mato, Daniel. (2000a). Des-fetichizar la "globalización": basta de reduccionismos, apologías y demonizaciones; mostrar la complejidad y las prácticas de los actores. Texto presentado en 
la II reunión del grupo de trabajo del CLACSO sobre cultura y transformaciones sociales en tiempos de globalización. Caracas, Venezuela, 9 al 11 de noviembre de 2000.

Mato, Daniel. (2000b). Introducción. En: Daniel Mato (Coord.). Estudios Latinoamericanos sobre cultura y cambio social en tiempos de globalización. Buenos Aires: Consejo Latinoamericano de Ciencias Sociales, CLACSO.

Monsonyi, Esteban. (1997). Neoliberalismo como antiantropología. Cuadernos Nuevo Sur, 10, 35-42.

Nader, Ralph. (1997). Neoliberalismo y globalización vistos desde el norte: el libre mercado y la declinación de la democracia. Cuadernos Nuevo Sur, 10, 72-81.

Reed, David. (Ed.). (1996). Ajuste estructural, ambiente y desarrollo sostenible. Caracas: Fondo Mundial para la Naturaleza. Cendes, Nueva Sociedad.

Rivadeneyra, Jorge. (1997). Globalización y globalizados. Cuadernos Nuevo Sur, 10, 26-31.

Rogalski, Michel. (1997). Globalización: de los beneficios a la coartada. Cuadernos Nuevo Sur, 10, 32-34.

Sampson, Edward. (1989). The challenge of social change for psychology. Globalization and psychology's theory of the person. American Psychologist, 44 (6) 914-921.

Serbin, Andrés. (1997). Globalización y sociedad civil en los procesos de integración. Nueva Sociedad, 147, 44-55.

Touraine, Alain. (1997). ¿Podemos vivir juntos? Iguales y diferentes. México D.F: Fondo de Cultura Económica.

Wiesenfeld, Esther. (2000). El lugar del/de la investigador/a en las investigaciones cualitativas: ¿Promesas (in) cumplidas?. FQS: Forum for Qualitative Studies, 1 (2). Accesible en: http://www.qualitative-research.net/fqs-texte/2-00/2-00wiesenfeld-s.htm

\section{Historia editorial}

Recibido: 21/07/2005

Primera revisión: 08/11/2005

Aceptado: 15/11/2005

\section{Formato de citación}

Wiesenfeld, Esther. (2006). El rescate de las comunidades en el marco de la Globalización. Athenea Digital, 9, 46-57. Disponible en http://antalya.uab.es/athenea/num9/wiesenfeld.pdf

Esther Wiesenfeld. Doctora en Psicología. Profesora Titular, Instituto de Psicología, Universidad Central de Venezuela. Autora de numerosas publicaciones. Miembro del Comité Editorial de varias 
revistas en psicología. Profesora invitada en universidades latinoamericanas y europeas. Disciplinas de interés: psicología ambiental y comunitaria. Líneas de trabajo: vivienda, habitat y procesos comunitarios; metodologías alternativas de investigación.

Este texto está protegido por una licencia Creative Commons.
Usted es libre de copiar, distribuir y comunicar públicamente la obra bajo las
siguientes condiciones:
Reconocimiento: Debe reconocer y citar al autor original.
No comercial. No puede utilizar esta obra para fines comerciales.
Sin obras derivadas. No se puede alterar, transformar, o generar una obra
derivada a partir de esta obra.
$\underline{\text { Resumen de licencia }}$
$\underline{\text { Texto completo de la licencia }}$

